This item is the archived peer-reviewed author-version of:

Title: The Coin Beneath the Crust: A Pilot Study of Coins from the Mediterranean Coast of Israel

Authors: A. Adriaens, M. Dowsett, E. Lehmann, Y. Farhi, J. Gunneweg, L.

Bouchenoire

In: A Holistic View on Qumran and the Dead Sea Scrolls, Gunneweg J., A. Adriaens, J. Dik (editors), Koninklijke Brill NV, Leiden, 2010, p.11-19.

To refer to or to cite this work, please use the citation to the published version:

A. Adriaens, M. Dowsett, E. Lehmann, Y. Farhi, J. Gunneweg, L. Bouchenoire, The coin beneath the crust: a pilot study of coins from the Mediterranean coast of Israel. In: A Holistic View on Qumran and the Dead Sea Scrolls, Gunneweg J., A. Adriaens, J. Dik (editors), Koninklijke Brill NV, Leiden, 2010, p.11-19.

\title{
The Coin Beneath the Crust: A Pilot Study of Coins from the Mediterranean Coast of Israel
}

\author{
A. Adriaens ${ }^{\text {a }}$, M. Dowsett ${ }^{\text {b }}$, E. Lehmann ${ }^{\text {c }}$, Y. Farhi ${ }^{\text {d }}$, J. Gunneweg ${ }^{\text {d }}$, \\ and L. Bouchenoire ${ }^{\mathrm{e}}$
}

\footnotetext{
${ }^{a}$ Department of Analytical Chemistry, Ghent University, Belgium, ${ }^{b}$ Department of Physics, Warwick University, UK, ${ }^{\mathrm{c}}$ Paul Scherrer Institute, Switzerland, ${ }^{\mathrm{d}}$ The Hebrew University, Jerusalem, ${ }^{\mathrm{e}} \mathrm{XMaS}$ beamline, ESRF, Grenoble, France
}

\begin{abstract}
We describe the application of synchrotron X-ray diffraction and neutron micro-tomography to three ancient bronze coins from the Mediterranean coast of Israel. Key questions are whether neutron tomography can contribute to the reading of features on corroded coins by allowing the corrosion crust to be stripped away in cyber-space using surface and volume rendering techniques, and to what extent this type of non-destructive measurement can inform possible conservation action. In addition, the X-ray diffraction experiments allow the main composition of the varicoloured surface corrosion to be deduced. We show that both X-ray and neutron data are of considerable help to numismatic study of the coins.
\end{abstract}

Keywords. Coins, Mediterranean coast of Israel, Neutron tomography, numismatics, synchrotron radiation $\mathrm{X}$-ray diffraction, corrosion, legibility 


\section{Introduction}

Ancient metal artefacts are usually found covered with a crust of corrosion in which some of the burial medium may be embedded. Since conversion of a metal to a corrosion product usually involves considerable swelling of the surface, relatively undamaged artefacts may be concealed beneath crusts of a millimeter or so in thickness.

Using three untreated coins with different degrees of corrosion we explore the hypothesis that neutron micro-tomography $(n-\mu \mathrm{T})$ might be used to obtain a complete 3 -D volumetric absorption map of the artefact in its as-found condition, and then volume and surface rendering techniques, common in tomography, could be used to distinguish between remaining metal and corrosion crusts. With sufficient resolution, it might even be possible to read the inscriptions on an artefact such as a coin, without disturbing the corrosion, or at least obtain more information as to the original nature of the artefact.

Since the crusts on the coins exhibited quite distinct patches of red, red-orange, greygreen and black corrosion, we decided to use synchrotron X-ray diffraction (SR-XRD) to look at the average composition of the top $10 \mu \mathrm{m}$ or so of each. Because of the speed inherent to the combination of synchrotron X-ray intensity $\left(\sim 10^{12}\right.$ photons $\left.\mathrm{s}^{-1}\right)$ and the use of an area detector, we could obtain a good survey of the coins in an hour of beam time. In future work, it may be possible to correlate the specific nature of the corrosion with the neutron absorption, and this is demonstrated to some extent here.

\section{Description of the coins}

The three coins (designated A, B and C) are discussed in chronological order, starting from the oldest. Photographs of the obverse (front side of the coin) and the reverse (back side of the coin) are shown in Figure 1 and Figure I* $^{*}$.

Coin B was found in the old city of Caesarea Maritima and dates to ca 610-641 CE. The coin is severely corroded, having a red-orange crust with patches of grey-green, and no obvious surface marks are preserved. Its visual identification is therefore somewhat conjectural. We suggest that it is a Byzantine coin minted under Heraclius. Coin A was found at the city of Átlit. It is in a better state of preservation. The corrosion is thin, but again red-orange with diffuse black and grey green regions. Nevertheless, it is easier to attribute to the Arab-Byzantine transition period of the mid seventh century CE. Coin $\mathrm{C}$ was also found at the city of Átlit. The corrosion on the two faces is different, and intermediate in thickness to that on coins A and C. One face is similar in appearance to the other coins, whilst the other is covered with a redder patina with a black ground. This may indicate that one side of the coin was burnt. The identification of this coin as Islamic is possible only due to the tomographic image (see 
later) which revealed the Arabic letters under the corrosion. A detailed numismatic report of the three coins can be found in Table 1. 


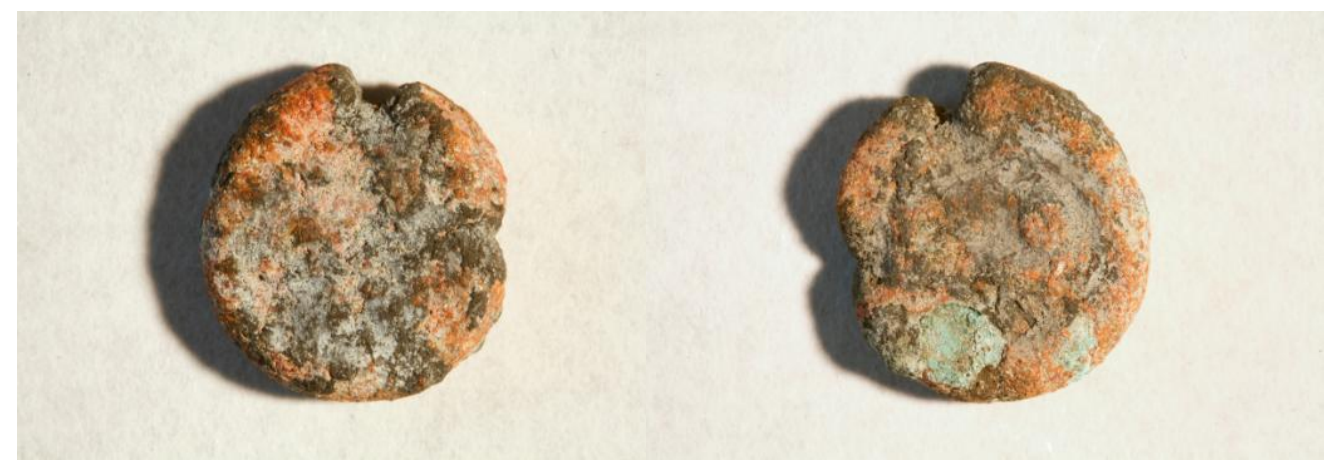

(a)

(b)

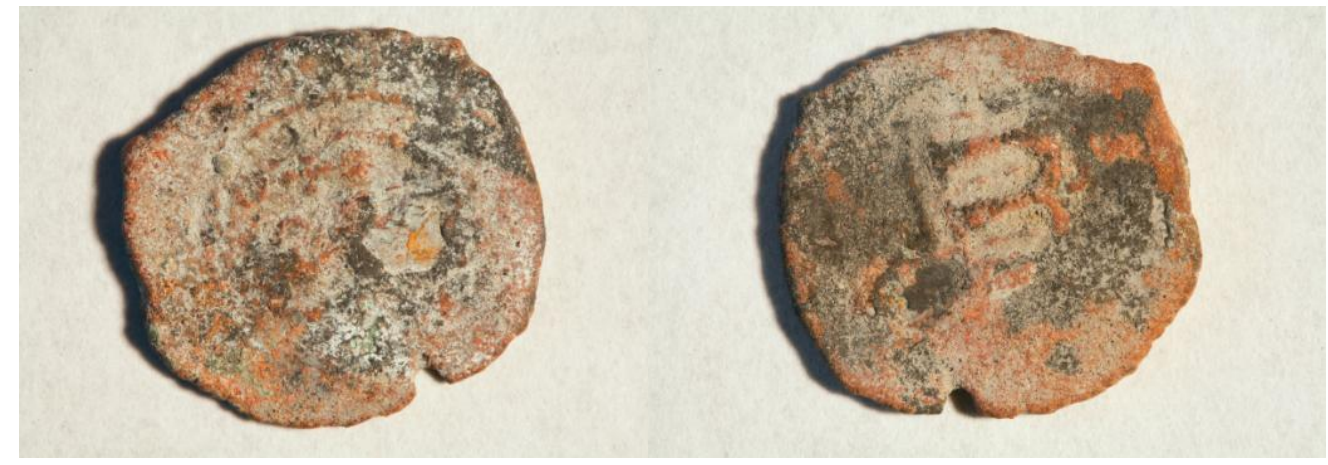

(c)

(d)

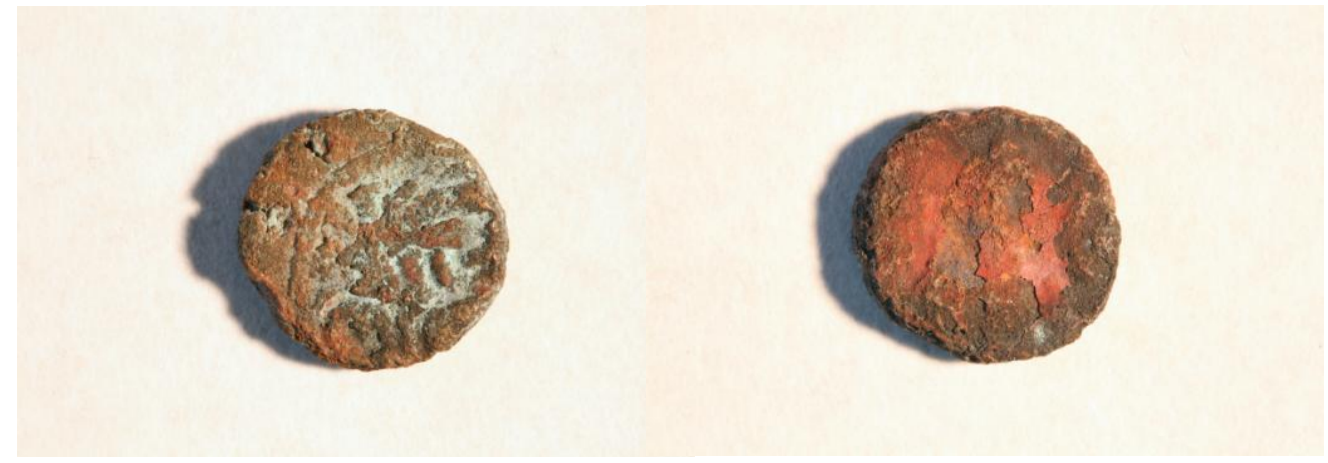

(e)

(f)

Figure 1. Photographs of the obverse (a, c and e) and reverse (b, d and $\mathrm{f}$ ) of respectively Coins B, A and C. A colour version is available in the plates (Figure I). 
Table 1. Numismatic data of coins B, A and C.

\begin{tabular}{|c|c|}
\hline Coin B & $\begin{array}{l}\text { Heraclius (610-641 CE)? } \\
\text { Obv. Bust of Heraclius to } 1 . \text { and bust of Heraclius Constantine to r. } \\
\text { No legends visible. } \\
\text { Rev. I+B (?), in exergue: }[\mathrm{A}] \Lambda[\mathrm{E} \Xi] \\
\text { Alexandria, } 613-618 \mathrm{CE} \text {. } \\
\text { Bronze, } \uparrow, 6.34 \text { gr., } 18 \mathrm{~mm} \text {. } \\
\text { Cf. Grierson P., Catalogue of the Byzantine Coins in the Dumbarton } \\
\text { Oaks Collection and in the Whittemore Collection, II, Part I, Phocas } \\
\text { and Heraclius (602-641). Washington, D. C. 1968. P. 334-335, nos. } \\
189.1-190 \text {. }\end{array}$ \\
\hline Coin A & $\begin{array}{l}\text { Arab Byzantine, } 7^{\text {th }} \text { Century (circa } 650 \mathrm{CE} \text { ). } \\
\text { Obv. Standing figure (emperor?) standing, facing; holding long } \\
\text { cross in r., and globus cruciger in } 1 ., \text { above the cross: O. Dotted } \\
\text { border. } \\
\text { Rev. Cursive } m \text { with cross above and pellets or stylized snakes (?) } \\
\text { between the uprights of the } \mathrm{m} \text {. Illegible legend in 1., no legends } \\
\text { visible in exergue. Dotted border. } \\
\text { Bronze, } \uparrow, 4.43 \text { gr., } 24 \mathrm{~mm} \text {. } \\
\text { Cf. Album S. and Goodwin T., Sylloge of Islamic Coins in the } \\
\text { Ashmolean, I, The Pre-Reform Coinage of the Early Islamic } \\
\text { Period. Oxford. } 2002 . \text { P. } 79 \text { (Type E); Goodwin T., Arab- } \\
\text { Byzantine Coinage. London. } 2005 . \text { P. } 40, \text { no. } 37 \text {. }\end{array}$ \\
\hline Coin $\mathrm{C}$ & $\begin{array}{l}\text { Islamic - Umayyad (post reform) or 'Abbāsid }\left(8^{\text {th }}-9^{\text {th }} \text { centuries CE) }\right. \\
\text { Obv. Uncertain Arabic inscription inside a circle. } \\
\text { Rev. No legends visible } \\
\text { Bronze, } \uparrow, 3.66 \text { gr., } 15 \mathrm{~mm} .\end{array}$ \\
\hline
\end{tabular}




\section{Experiments}

X-ray diffraction experiments were performed at XMaS (Station BM28, European Synchrotron Radiation Facility, Grenoble, France). At this station, a beam with a wavelength of $1.5498 \AA$ and with dimensions of $1 \mathrm{~mm} \times 200 \mu \mathrm{m}$ was used. A 2D Mar CCD 165 detector (Mar USA Inc., Evanston, IL, USA) was used to record the diffraction patterns. The angle of the camera to the beam was $35^{\circ}$ in order to be able to acquire the signals $2 \Theta$ values of $15^{\circ}$ and $75^{\circ}$. Under these conditions, the diffraction centre is outside the field of view of the camera, and the camera plane intersects the diffraction cones at an angle to produce elliptical "rings". The images were processed using a new software program, esaProject (C) EVA Surface Analysis), which was developed to extract spectra from such images.

Neutron $\mu \mathrm{T}$ experiments were performed at the spallation neutron source SINQ, located at the Paul Scherrer Institute, using the ICON beam line for cold neutrons. The advantage of cold neutrons for imaging purposes is their higher contrasts for most of the sample materials and the higher detection probability. A pixel size of $13.5 \mu \mathrm{m}$ was used. The set-up is shown in Figure 2.

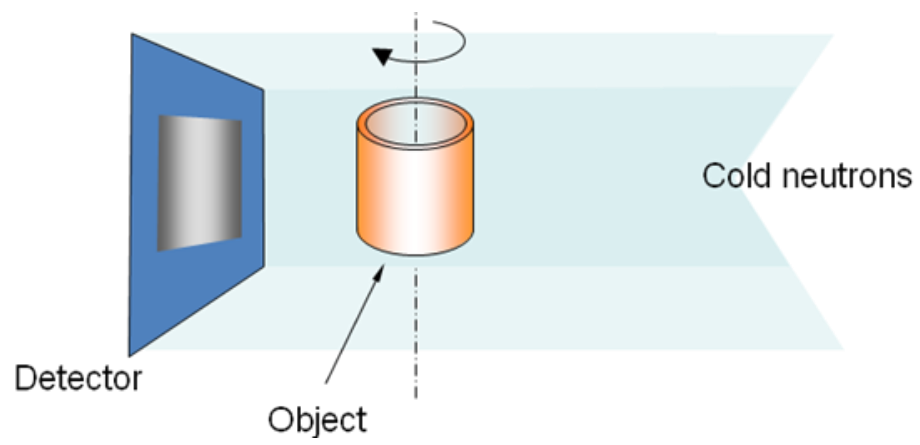

Figure 2. Principle of cold neutron tomography

\section{X-ray diffraction data}

The three coins show mainly the presence of black and red to orange corrosion products which are a mixture of cuprite $\left(\mathrm{Cu}_{2} \mathrm{O}\right)$ and tenorite $(\mathrm{CuO})$ in various proportions - cuprite being responsible for the red and tenorite for the black colouration. A typical spectrum is shown in Figure 3a. The grey-green patches on coins $\mathrm{B}$ and $\mathrm{C}$ prove to be a mixture of cuprite, tenorite and paratacamite (a copper hydroxychloride), as is shown in Figure $3 b$. 

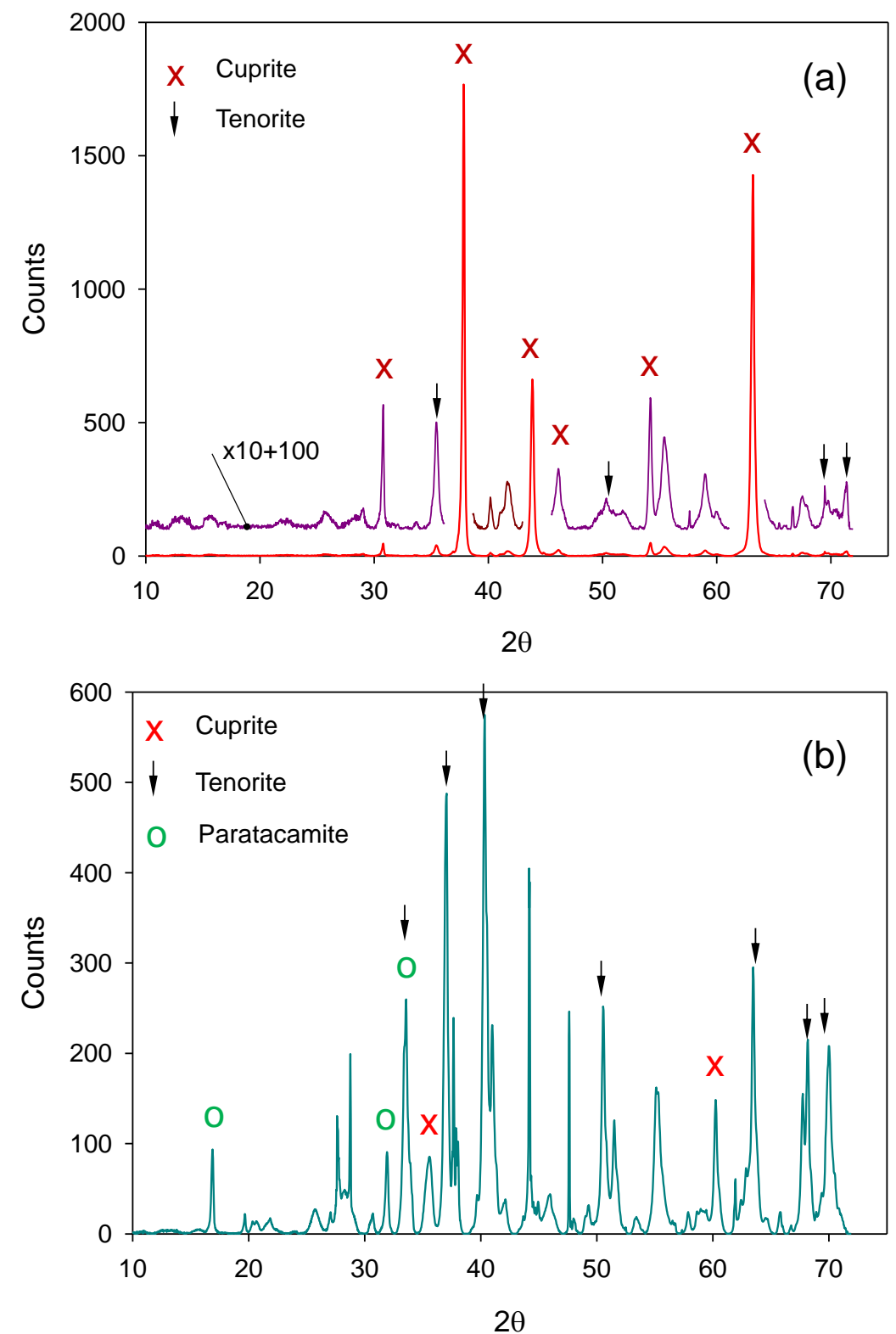

Figure 3: (a) Showing XRD characteristic of the orange-red and black regions on all 3 coins and (b) the grey-green regions on coins $\mathrm{B}$ and $\mathrm{C}$.

\section{Neutron tomography data}

Photographs and near-surface neutron tomography images for the observe and reserve of the three coins are shown in Figure 4 and Figure II $^{*}$. In the case of coin B (top row) the rendered surface in the tomography passes beneath the paratacamite regions at the bottom of the photograph (third pane from the left). These appear as craters in the 
tomographic surface in the right hand pane. The rendering also passes beneath the tenorite, revealing surface relief filled by the oxide. This is a clear demonstration that the neutron stopping power of the different corrosion products is sufficiently different for them to be distinguishable. A similar effect is seen for coin A (second row), with details of the embossing on the coin being more prominent in the rendered surfaces from the neutron tomography (second and last pane) in comparison with the photographs (first and third pane). This is again because the rendered surface passes beneath the corrosion layer. Of course, photography using controlled illumination, filters and polarized light might achieve a similar effect, but as we show below, the distinction between corrosion and metal can be applied to any part of the volume of the coin to reveal internal features non destructively. The third row shows the comparison between photographs and rendered surfaces for coin $\mathrm{C}$. Here, the improvement in readability is a distinct aid to identification, and even on the reverse side some evidence of embossing is revealed, albeit unidentifiable.
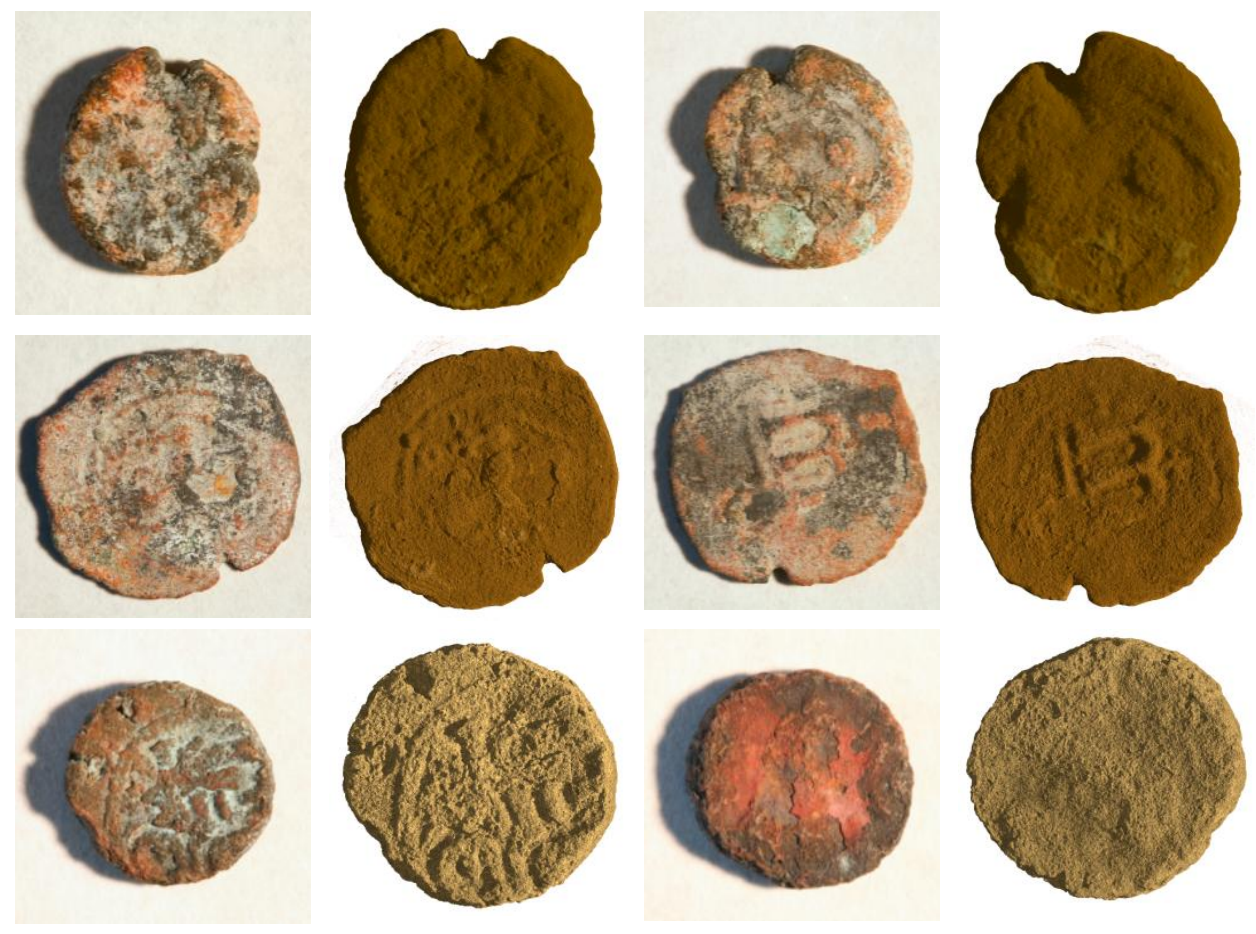

Figure 4. Top row: photographs and neutron tomography images of the surface of obverse (left) and reverse (right) of Coin B. Middle row: idem for Coin A. Bottom row: idem for Coin C. A colour version is available in the plates (Figure II). 
(a)
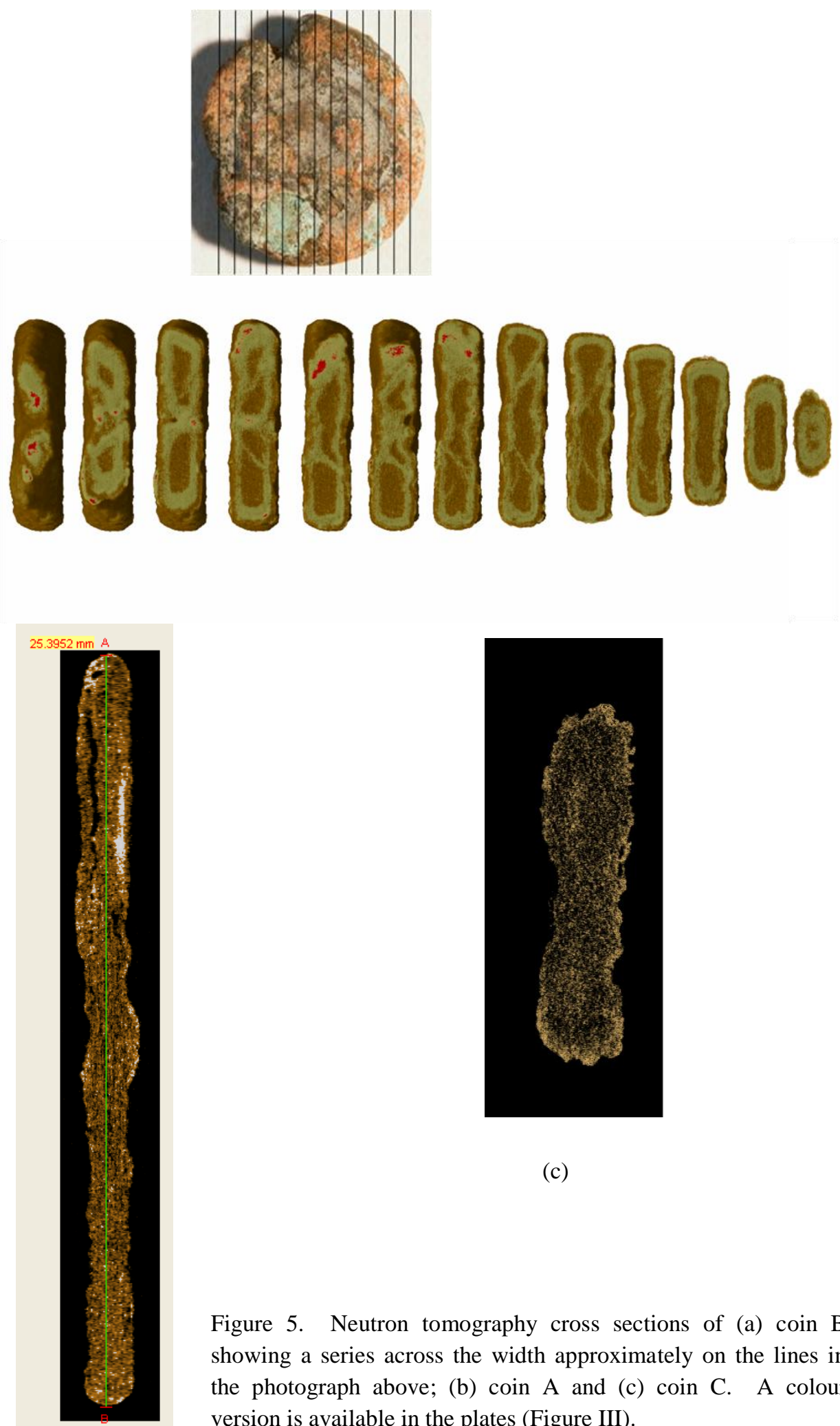

(c)

Figure 5. Neutron tomography cross sections of (a) coin B showing a series across the width approximately on the lines in the photograph above; (b) coin A and (c) coin C. A colour version is available in the plates (Figure III). 
Figure 5 and Figure III $^{*}$ show neutron tomography images from inside the three coins. For coin B a series is shown from one end of the coin to the other. The set of images shows a different attenuation for different regions, which is visualized by the different colours: brown, grey and red. It is clear that the coin has corroded from within and without, with internal corrosion occurring in cracks and voids causing the structure to swell. The images show that there is hardly any metal remaining.

The cross section of coin A shows the presence of two different materials and several cracks inside the coin. Some of these show evidence of developing corrosion - perhaps a precursor to the fate of coin $\mathrm{B}$. Coin $\mathrm{C}$ on the other hand is composed of a quite homogenous material as judged by the neutron stopping power, although with a different porosity through the coin: the inner part of the coin $\mathrm{C}$ seems to be quite porous, whereas the outer part does not.

\section{Conclusions}

In this work we have examined three corroded coins which originate from the Mediterranean coast in Israel. Numismatic data show that they are from the $6^{\text {th }}$ to the $9^{\text {th }}$ century CE. The corrosion crusts mainly constitute tenorite and cuprite with patches of paratacamite. Neutron tomography data are able to visualize the inner structure of the three coins showing the heterogeneity and the presence of cracks and different chemical compounds as also indicated by the XRD data. Surface rendering of volumes in a particular range of neutron stopping power reveal the metal surface and can increase the readability of the markings (if any remain). In particular, for coin C, the neutron tomography data contribute to the identification of the coin through its surface inscription .

\section{Acknowledgements}

The authors would like to thank Melanie Salque in helping with the interpretation of the neutron tomography images and Roger Gibbons for the photography of the coins. This work would not have been possible without the support of COST Actions G8 and D42, and EVA Surface Analysis (UK).

\footnotetext{
${ }^{*}$ Figures numbered in roman are in colour and can be found in the plates.
} 